\title{
Monitoring latent tuberculosis infection diagnosis and management in the Netherlands
}

\author{
Connie G.M. Erkens ${ }^{1}$, Erika Slump ${ }^{2}$, Maurits Verhagen ${ }^{3}$, Henrieke Schimmel ${ }^{2}$, \\ Gerard de Vries ${ }^{1,2}$, Frank Cobelens ${ }^{1,4}$ and Susan van den Hof ${ }^{1,4}$
}

\author{
Affiliations: \\ ${ }_{1}^{1}$ KNCV Tuberculosis Foundation, The Hague, The Netherlands. \\ ${ }^{2}$ Centre for Infectious Disease Control, National Institute for Public Health and the Environment, Bilthoven, \\ The Netherlands. \\ ${ }^{3}$ Municipal Public Health Service (GGD) Limburg-Noord, Roermond, The Netherlands. \\ ${ }^{4}$ Dept of Global Health, Academic Medical Center, Amsterdam Institute for Global Health and Development, \\ Amsterdam, The Netherlands.
}

\section{Correspondence:}

Connie G.M. Erkens, KNCV Tuberculosis Foundation, PB 146, 2501 CC The Hague, The Netherlands.

E-mail: Connie.erkensakncvtbc.org

ABSTRACT Targeted diagnosis and treatment of latent tuberculosis (TB) infection (LTBI) among persons with a high risk of exposure to TB or of developing TB when infected has been performed and monitored routinely in the Netherlands since 1993. We describe trends in target groups, diagnostic methods and treatment regimens, and explore determinants for treatment initiation, treatment completion and adverse events.

In total, 37729 persons were registered with LTBI from 1993 to 2013, of whom 28931 (77\%) started preventive treatment; $82 \%$ of those completed preventive treatment and $8 \%$ stopped preventive treatment due to adverse events. Two-thirds of the notified cases were detected through contact investigation.

Increasing numbers of persons with immunosuppressive disorders, elderly persons and foreign-born persons were notified in recent years, due to policy changes and the introduction of the interferon- $\gamma$ release assay. Children (96\%) and the immunosuppressed (95\%) were more likely to start preventive treatment. Children (93\%) were also more likely to complete preventive treatment, as were persons treated with rifampicin or rifampicin/isoniazid regimens ( $91 \%$ and $92 \%$, respectively). The latter groups were also $40 \%$ less likely to stop preventive treatment due to adverse events.

Under these operational conditions, the estimated risk reduction on incident $\mathrm{TB}$ in the target population for LTBI management is $40-60 \%$.

@ERSpublications

Systematic monitoring and evaluation enables successful management of latent TB infection http://ow.ly/X6D2A

Editorial comment in: Eur Respir J 2016; 47: 1327-1330.

This article has been revised according to the correction published in the January 2017 issue of the European Respiratory Journal.

This article has supplementary material available from erj.ersjournals.com

Received: Aug 232015 | Accepted after revision: Jan 11 2016 | First published online: Feb 252016

Conflict of interest: None declared.

Copyright OERS 2016 


\section{Introduction \\ Background}

In May 2014, the World Health Organization (WHO) launched the End TB Strategy to step up efforts for the elimination of tuberculosis (TB) worldwide [1]. For low-incidence countries, defined as countries with a TB incidence of less than 10 per 100000 population, WHO and the European Respiratory Society (ERS) developed a Framework towards TB elimination [2,3] and WHO developed guidelines for latent TB infection (LTBI) management in low TB burden countries [4]. One of the key interventions of the Framework is screening for LTBI in populations at high risk for TB and providing preventive treatment for those infected. The Netherlands already reached the status of a low TB incidence country in the early 1980s. Since then, TB notification rates have declined steadily to 4.9 per 100000 persons in $2014 ; 73 \%$ of patients were foreign-born in 2014, and TB notification rates were 1.5 and 33.3 cases per 100000 in the Dutch-born population and the foreign-born population, respectively.

Targeted LTBI screening of specific high-risk groups has been a pillar of TB control for decades in the Netherlands in addition to active TB case finding, such as radiological screening of new immigrants and asylum seekers [5] (table 1). Municipal Public Health Services (MPHSs) carry out LTBI screening and treatment among $\mathrm{TB}$ contacts, (health) professionals frequently exposed to $\mathrm{TB}$ and travellers to $\mathrm{TB}$ endemic areas. LTBI screening of target groups such as immunocompromised persons is mostly done in a clinical setting. The diagnosis of LTBI is based on 1) the tuberculin skin test (TST) or interferon- $\gamma$ release assay (IGRA), 2) assessment of the likelihood of (recent) TB exposure and 3) exclusion of active TB. Screening algorithms differ between specific age and target groups (online supplementary table S1). IGRA was first used in the Netherlands around 2005. In 2010, the national guidelines for LTBI diagnosis recommended a two-step approach using IGRA in those with a TST reaction $\geqslant 5 \mathrm{~mm}$ (online supplementary table S1) [6]. Persons diagnosed with LTBI are offered preventive treatment or 2 years of radiological follow-up, depending on their probability of developing $\mathrm{TB}$, contra-indications for preventive treatment and willingness to start preventive treatment. Before 2000, the preferred preventive treatment regimen was 6 months of isoniazid. From 2000 onwards, shorter regimens were increasingly used and

TABLE 1 Major policy changes in latent tuberculosis (TB) infection (LTBI) diagnosis and treatment 1993-2013

$\begin{array}{ll}\text { Period (revision) Policy (guideline) Target group } & \text { Tre }\end{array}$

1993-2004 Source and contact investigation

Healthcare workers and other professional contacts of risk groups

Travellers to TB endemic areas ${ }^{\#}$

Pre-BCG examination

2004

2004

2005

2008

2009

2010

2013
Preventive treatment of persons with inactive fibrotic lesions with no history of previous TB treatment Screening before treatment with TNF- $\alpha$ inhibitors Source and contact investigation

\section{Screening HIV-infected persons}

Introduction of short-course preventive treatment regimen with 3 months of rifampicin/isoniazid and 4 months of rifampicin (LTBI treatment guideline) IGRA officially included in diagnostic algorithm for LTBI

Travellers to TB endemic areas $\#$, "
Close contacts, excluding persons born before 1945 , immigrants from endemic areas ${ }^{\#}, B C G$-vaccinated persons

Healthcare workers and other professional contacts of risk groups (such as staff asylum reception centres, prison staff), pre-exposure and periodic screening

Long-term travellers (>3 months), pre- and post-exposure

Children aged 6 months to 12 years with a parent from an endemic area (children aged 6-12 months only when travelled in endemic areal

New immigrants arriving from TB endemic countries

Patients starting TNF- $\alpha$ inhibitor treatment

Expansion of eligibility for LTBI screening to persons vaccinated with BCG during infancy and immigrants having lived $<12$ years in TB endemic areas HIV-infected persons

All persons eligible for preventive treatment

BCG-vaccinated persons, close TB contacts originating from TB endemic areas, persons born before 1945

Post-exposure for travellers with high risk of exposure to TB determined by: risk setting, length of stay and TB incidence

BCG: bacille Calmette-Guerin; TNF: tumour necrosis factor; IGRA: interferon- $\gamma$ release assay. ${ }^{\#}$ : endemic area defined as estimated TB incidence according to the World Health Organization of more than 50 per 100000 population; ${ }^{\text {I }}$ : pre-exposure examination of travellers was abolished and post-exposure screening only for specified high-risk travellers. 
4 months of rifampicin only or 3 months of rifampicin/isoniazid has been recommended in national guidelines since 2008 .

In this paper, we describe the LTBI recording and reporting tool, and the results of 21 years of LTBI monitoring and evaluation, focusing on trends in target groups for LTBI screening and preventive treatment regimens used. In addition, we examine the cause of death during preventive treatment, and explore risk factors associated with preventive treatment initiation, preventive treatment completion and preventive treatment discontinuation related to the occurrence of adverse events and estimated the risk reduction by preventive treatment for active TB among the population identified with LTBI.

\section{LTBI monitoring system}

The MPHSs register LTBI cases in the Netherlands Tuberculosis Register (NTR). The NTR is an anonymised nationwide web-based case-based surveillance system for TB and LTBI. TB notification is mandatory and LTBI is reported on a voluntary basis. The system started in 1993 as a paper case-based registry for both TB and LTBI cases. It captures LTBI cases newly diagnosed by or reported to the MPHSs. Data recorded include patients' demographic characteristics, target group, diagnostic method and patient management (preventive treatment regimen or radiological follow-up), completion of preventive treatment, and reason for interrupting preventive treatment (adverse events, development of active TB or nonadherence). Reasons for nonacceptance of preventive treatment are not captured. Data are collected in two stages: demographic and diagnostic data in stage 1 , and treatment outcome data in stage 2 . For each stage, dedicated data managers validate the data by checking for internal consistency and completeness. Only data with a validated stage 1 status are used to determine the number of cases reported and only treatment outcome results with a validated stage 2 status are used to determine the treatment outcome results.

In 2005, the case record form (CRF) for notification was split in separate CRFs for TB and LTBI. Both forms were integrated with the central web-based register for infectious disease surveillance "OSIRIS", hosted by the National Institute for Public Health and the Environment (RIVM). The content of the LTBI CRF was revised. At that time, it was observed that the case definition of LTBI to be reported was not clear: some MPHSs were only reporting cases starting preventive treatment, while others would also report newly diagnosed cases considered as "old or remote" infections not eligible for preventive treatment. A new case definition of LTBI was agreed, strictly based on the eligibility for preventive treatment according to the national guidelines. This includes cases with 1$)$ a high likelihood of recent infection ( $<2$ years ago), 2) severe immunosuppressive disorders (e.g. HIV infection), 3) pulmonary fibrotic lesions consistent with active $\mathrm{TB}$ in the past and without adequate treatment, and 4) planned immunosuppressive therapy (tumour necrosis factor (TNF)- $\alpha$ antagonists/organ transplantation). Child $\mathrm{TB}$ contacts and immunocompromised TB contacts receiving primary chemoprophylaxis are not considered as reportable LTBI cases. They are reported if they show a test conversion later.

Additional categories for target groups of LTBI screening which previously were recorded under "other" were introduced in the CRF: persons with severe immunosuppressive disorders or before use of immunosuppressive medication, persons with inactive intra-thoracic fibrotic lesions and IGRA as the diagnostic method. The nature of adverse events during preventive treatment was no longer recorded in the new CRF for LTBI. Both TB and LTBI data are analysed and reported annually in the national surveillance report and made available for use by interested parties through a publicly accessible website (www.tbc-online.nl/eng/).

\section{Methods}

The NTR registration committee approved the use of data from the NTR of all LTBI cases notified in the period 1993-2013 for the purpose of the study. In addition, we collected information from the MPHSs through a short questionnaire on the cause of death for patients who were reported to have died during preventive treatment.

We conducted a descriptive analysis of the population characteristics and trends in treatment acceptance and treatment completion over time. Trend analysis was stratified according to country of origin and target groups for LTBI management.

Target groups were classified reflecting the likelihood of recent exposure: contact investigation, post-exposure screening (screening of at-risk professionals and travellers), pre-exposure screening (at-risk professionals and travellers and pre-bacille Calmette-Guerin (BCG) vaccination) and screening related to immunosuppression/ before immunosuppressive therapy. The latter group includes both immunocompromised persons and immunocompetent patients. For the purpose of the analyses, an adverse event was defined as any adverse event leading to discontinuation of preventive treatment. 
We conducted a multivariable analysis with logistic regression. We determined the factors independently associated with 1) treatment initiation, 2) treatment completion and 3) (discontinuation due to) adverse events with multivariable regression analysis. A priori confounders (age, sex, country of origin, period) were fixed in the multivariable model. Other variables yielding a $\mathrm{p}$-value $<0.2$ in the univariable analysis were included in the model, and the most parsimonious model was selected by backward elimination guided by the change in log likelihood and coefficients of successive models. Statistical analysis was performed in SPSS version 22.0 (SPSS, Chicago, IL, USA).

The risk reduction of preventive treatment for active $\mathrm{TB}$ was calculated as the product of the rate of preventive treatment initiation and completion in the period 2009-2013 and the reported efficacy of the different preventive treatment regimens (60-90\%) [7].

\section{Results \\ Trends and characteristics of cases notified with LTBI}

In total, 37729 persons were diagnosed and reported with LTBI from 1993-2013. The annual number of registered LTBI cases increased between 1993 and 1999 from 1356 to 2435 and dropped to the level of 1993 from 2010 onwards (figure 1). After 2005, the number decreased for cases starting treatment as well as for those not starting treatment. The number of reported Dutch-born LTBI cases increased until 2002, and then decreased steeply between 2005 and 2013 from 1870 to 690 cases, while the number of reported foreign-born LTBI cases increased from $271(13 \%)$ to $655(49 \%)$ in the same period. At the same time, the age (mean \pm SD) of those identified with LTBI increased from $27.3 \pm 10.1$ years in 1993 to $37.0 \pm 16.2$ years in 2013. After 2005, IGRA was increasingly used for the diagnosis of LTBI, up to $83 \%$ in the cases notified in 2013 (figure 1).

\section{Preventive treatment initiation}

In total, 28931 of 37729 (77\%) reported LTBI cases started preventive treatment (figure 2). During 1993-2013, the percentage of persons with LTBI receiving preventive treatment dropped from $97 \%$ to $65 \%$ in 2005 , steadily increased again to $79 \%$ in 2011 and dropped again to $71 \%$ in 2013 . Overall, the main indication for LTBI screening among LTBI cases initiating preventive treatment was contact investigation (61\%, annual range 54-71\%), followed by pre-exposure evaluation (19\%, annual range 7-28\%). Until 2005, the number of LTBI cases initiating preventive treatment increased in all target groups. After 2005, the absolute number of cases declined in all target groups, except in the new target groups of persons with immunocompromised conditions and persons with fibrotic lesions.

Persons registered from 1993 to 1996 (93\%), children < 15 years (96\%), immunocompromised persons (95\%), persons with fibrotic lesions (83\%) and persons identified through contact investigation (79\%) were more likely to start preventive treatment. After multivariable adjustment, having received preventive treatment was associated with registration in the period 1993-1996 or 2009-2013, age <15 years, female sex, contact investigation, immune disorder or fibrotic lesions as reason for testing and negative BCG vaccination status. Being tested with IGRA alone was negatively associated with initiating preventive treatment (table 2).

\section{Preventive treatment completion}

In total, 27748 of 28931 persons (96\%) initiated on preventive treatment had a registered and validated result of preventive treatment. In addition to the 1183 cases on preventive treatment without a validated registered result of preventive treatment, 2192 persons with a validated result were lost to follow-up, i.e. a total of $12 \%$ of those initiated on preventive treatment.

Of 27748 persons with a valid result of preventive treatment, $85 \%$ completed the treatment, i.e. $82 \%$ of the total registered cases on preventive treatment. Children (93\%), professionals (91\%), travellers post-exposure (93\%), and persons with fibrotic lesions (93\%) were more likely to complete the treatment. The completion rates varied over time. After the multivariable analysis, diagnosis in 1993-1996 or 2009-2013, age $<15$ years, male sex, Dutch born, rural residence, target groups immunosuppression, professional at-risk, traveller and fibrotic lesions, and rifampicin-containing regimens were associated with treatment completion (table 3). Compared with persons treated with 6-9 months of isoniazid, the adjusted odds ratios for completing preventive treatment for persons treated with 4 months of rifampicin or a combination of 3-4 months of rifampicin/isoniazid were 1.7 (95\% CI 1.3-2.2) and 1.7 (95\% CI 1.4-2.0), respectively.

\section{Adverse events}

In total, $2105(7.6 \%)$ persons discontinued preventive treatment because of adverse effects. The nature of adverse events was only registered during the period 1993-2004. Of 1392 persons who stopped preventive treatment during this period due to adverse events, $33 \%$ had hepatic dysfunction, $10 \%$ neurological dysfunction, $11 \%$ psychological dysfunction, $10 \%$ allergy, $1 \%$ visual dysfunction, $1 \%$ arthralgia, $21 \%$ nonspecified adverse events and $12 \%$ unknown. 
$17(0.1 \%)$ persons were reported to have died during preventive treatment. For seven of these, of whom five registered before 2005, we were unable to retrieve information on the cause of death. For the remaining 10 , the cause of death was not related to the preventive treatment according to the MPHSs. Two persons committed suicide with an overdose of isoniazid and eight persons died from cardiac events, malignancies or other specified nonpreventive treatment-related causes.

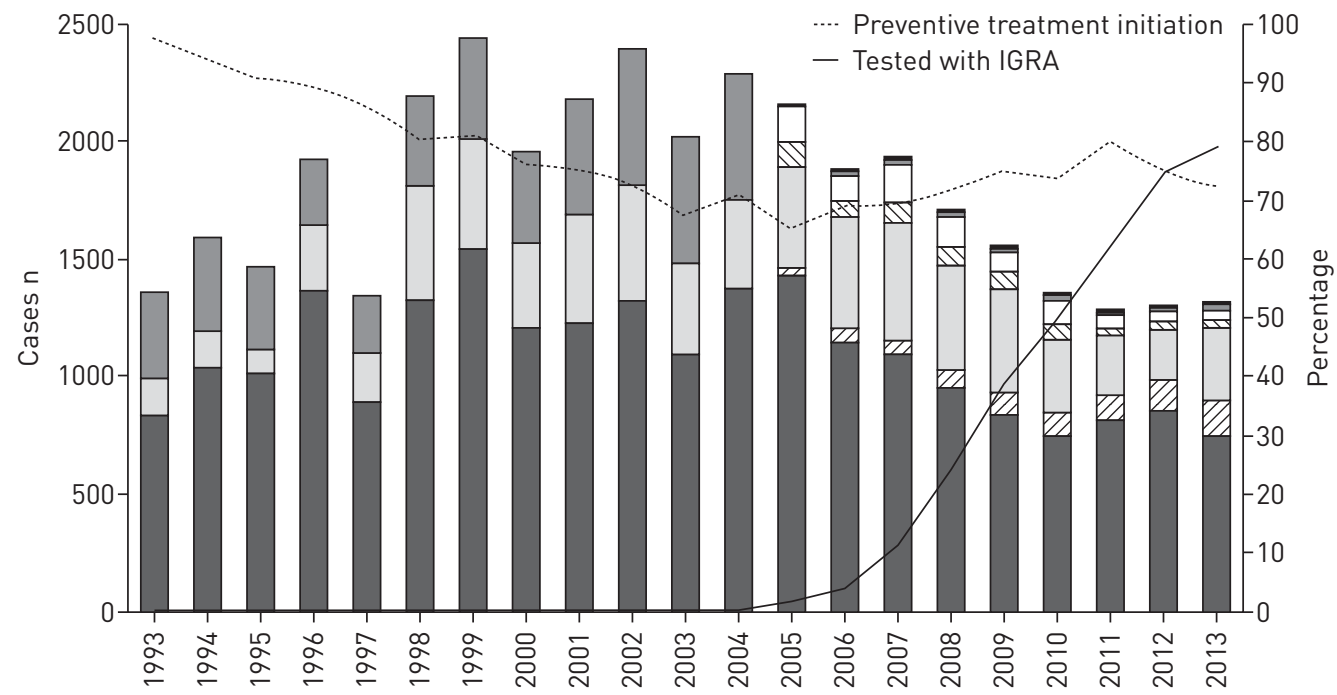

Contact investigation

$\square$ Immunosuppression\#

Pre-exposure examination

N Professional exposure\#

$\square$ Traveller from endemic area\#

Other post-exposure

Fibrotic lesions \#

FIGURE 1 Number of cases with latent tuberculosis infection by target group for screening, and percentage screened with interferon- $\gamma$ release assay (IGRA) and preventive treatment initiation (1993-2013). \#: reported since 2005 .

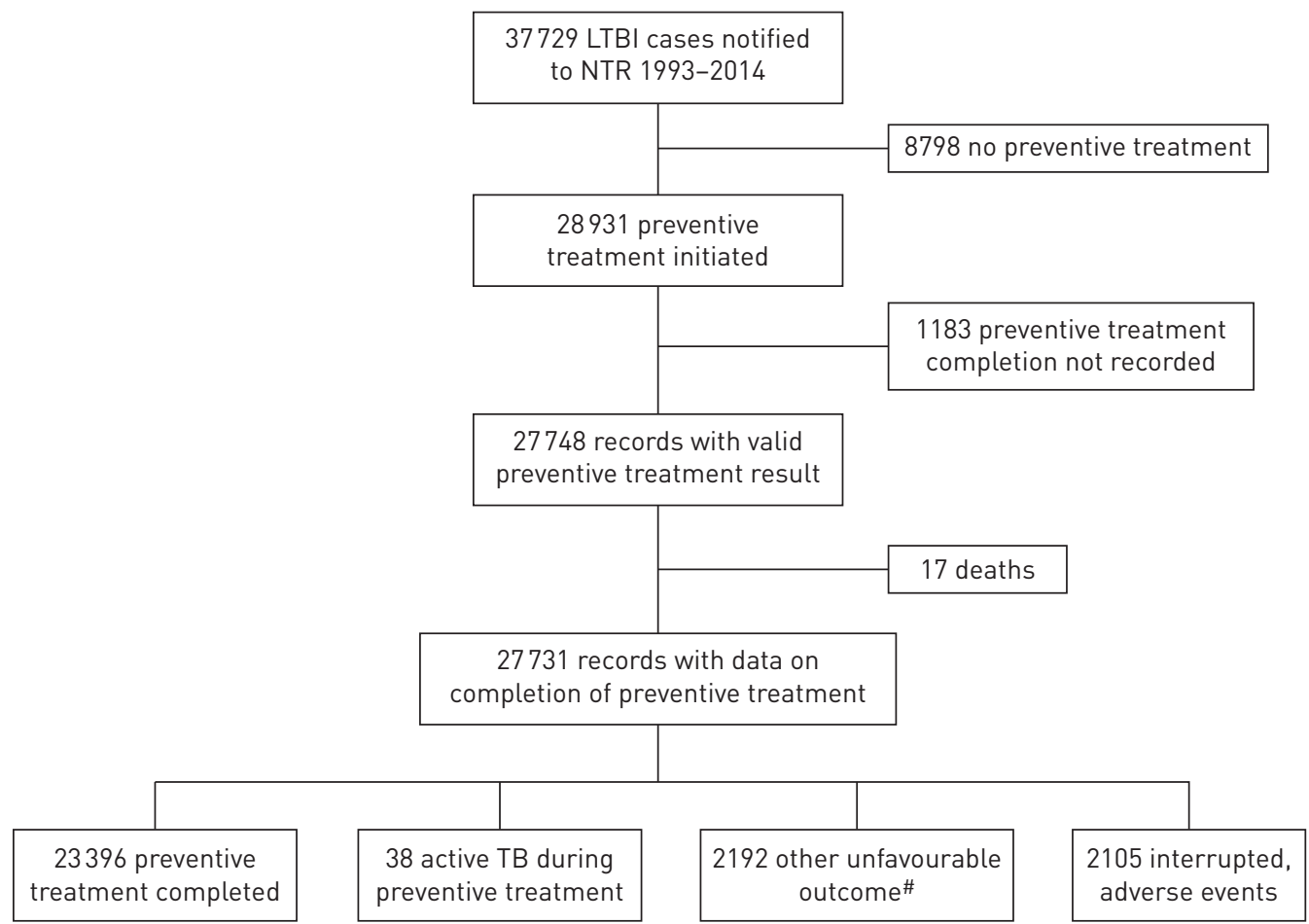

FIGURE 2 Flowchart for risk factor analysis in latent tuberculosis (TB) infection (LTBI). NTR: Netherlands Tuberculosis Register. " : other unfavourable outcome is defined as treatment interrupted by patient, transfer out or outcome unknown. 
TABLE 2 Univariable and multivariable analysis of determinants of preventive treatment initiation in persons notified with latent tuberculosis (TB) infection initiated on preventive treatment

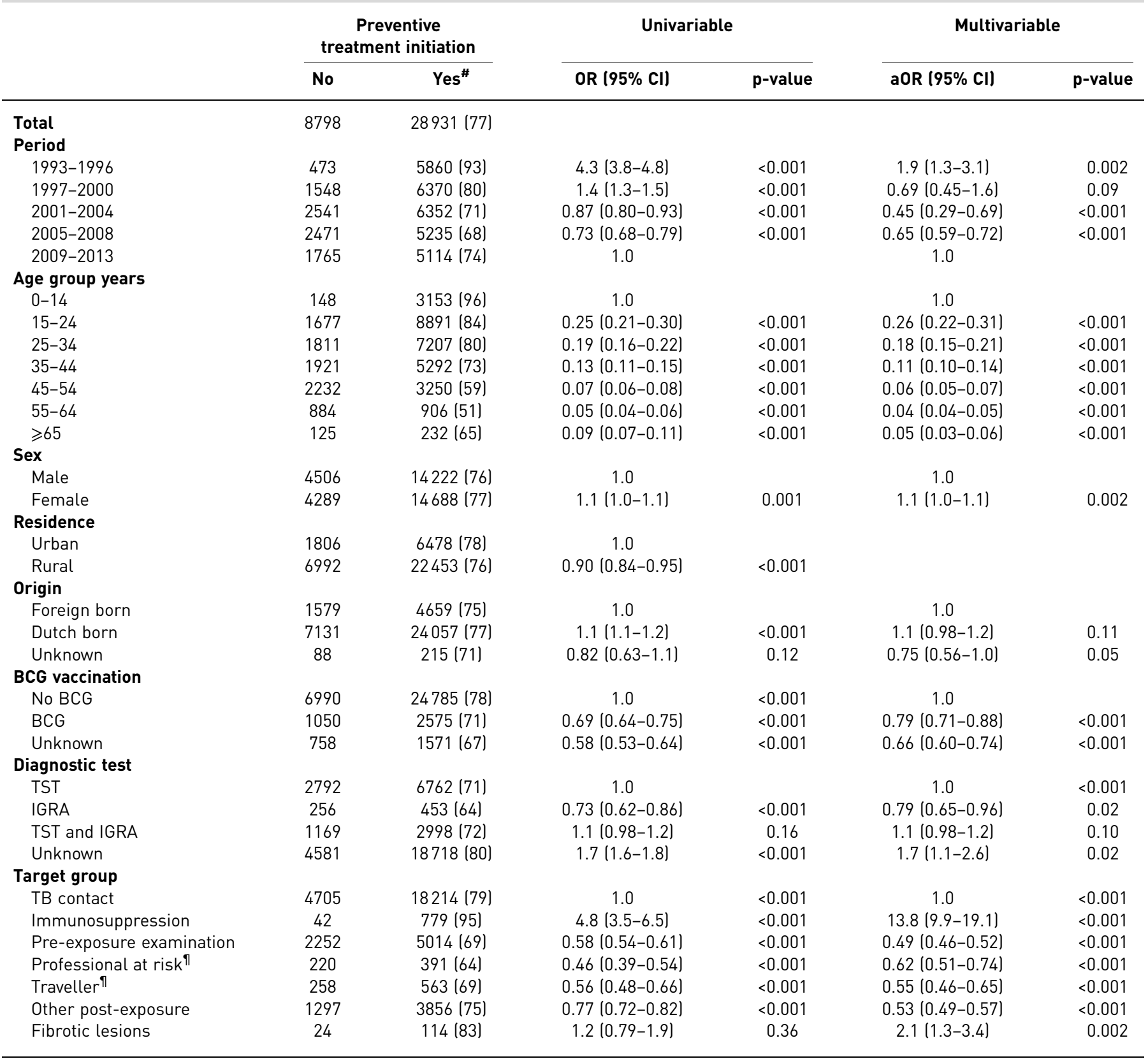

Data are presented as $\mathrm{n}$ or $\mathrm{n}(\%)$, unless otherwise stated. OR: odds ratio; aOR: adjusted OR; BCG: bacille Calmette-Guerin; TST: tuberculin skin test; IGRA: interferon- $\gamma$ release assay. " : percentage treatment initiated of row total given in parentheses; ${ }^{\text {I: }}$ post-exposure.

Factors associated with interruption of treatment due to adverse events were female sex, Dutch born, rural residence and isoniazid monotherapy as preventive treatment regimen. Diagnosis from 1997 to 2008, age $<5$ years and rifampicin-containing regimens were associated with a lower likelihood of interruption of treatment due to adverse events (table 4). After controlling for these factors and compared with persons treated with 6-9 months of isoniazid, the adjusted odds ratio of stopping preventive treatment due to adverse events was lowest among persons treated with 4 months of rifampicin and 3-4 months of rifampicin/isoniazid: 0.63 (95\% CI 0.44-0.90) and 0.58 (95\% CI 0.45-0.74), respectively.

Risk reduction for developing TB in those found with LTBI

In the period 2009-2013, 74\% of eligible persons started preventive treatment and $90 \%$ completed preventive treatment. Assuming an estimated effectiveness of preventive treatment regimens between $60 \%$ 
TABLE 3 Factors associated with completion of preventive treatment

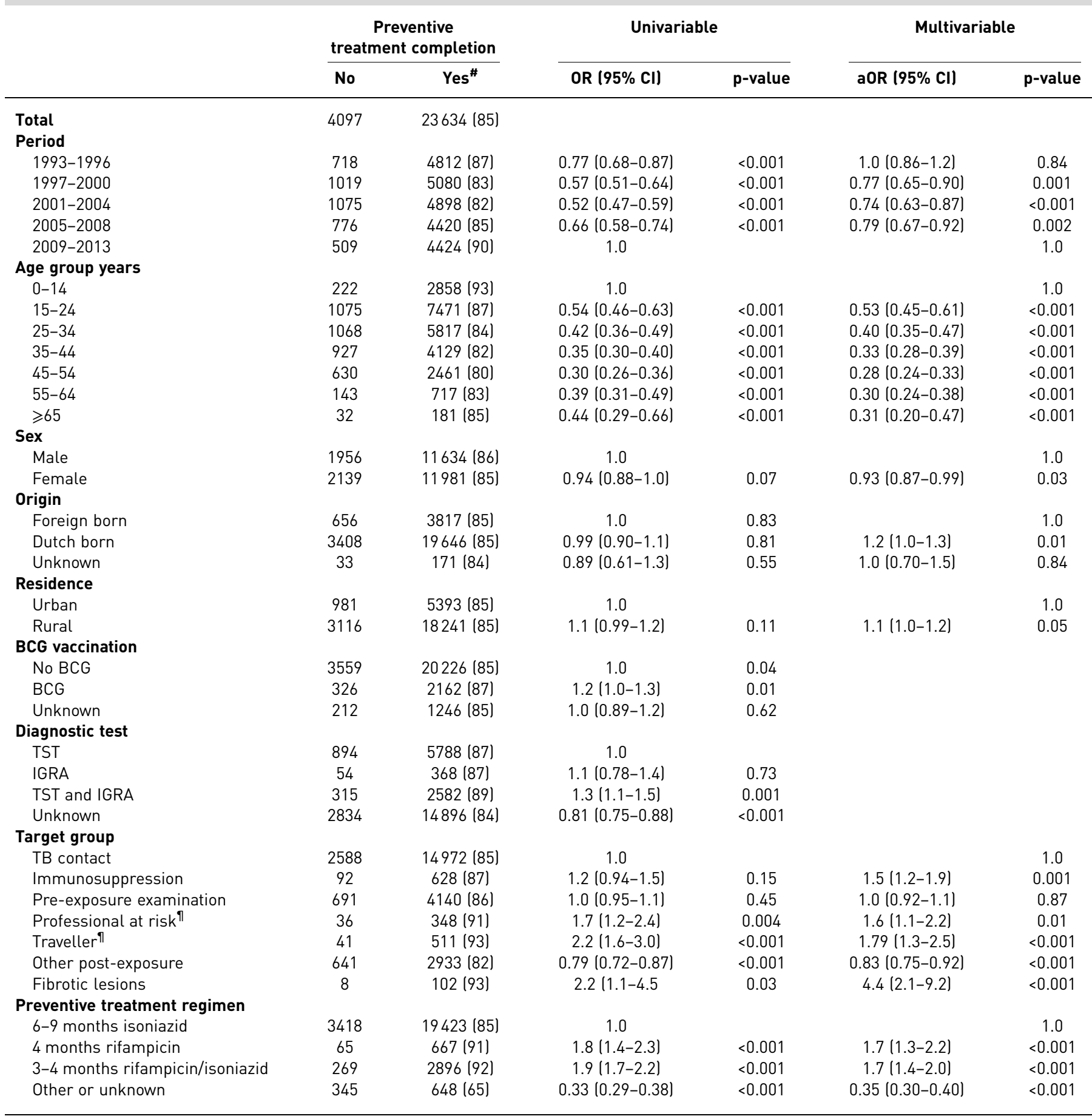

Data are presented as $\mathrm{n}$ or $\mathrm{n}(\%)$, unless otherwise stated. OR: odds ratio; aOR: adjusted OR; BCG: bacille Calmette-Guerin; TST: tuberculin skin test; IGRA: interferon- $\gamma$ release assay; TB: tuberculosis. ${ }^{\#}$ : percentage completed of row total given in parentheses; ${ }^{\text {ๆ: }}$ post-exposure.

and $90 \%$, the risk reduction of preventive treatment for active TB among the total population identified with LTBI is between $40 \%$ and $60 \%$.

\section{Discussion}

During 21 years of programmatic LTBI management in the Netherlands, 37729 persons were registered with LTBI; of those, $77 \%$ started preventive treatment. In total, $82 \%$ of all reported cases on treatment were known to have completed the treatment. Two-thirds of the notified LTBI cases were detected through contact 
TABLE 4 Factors associated with discontinuing treatment due to adverse events

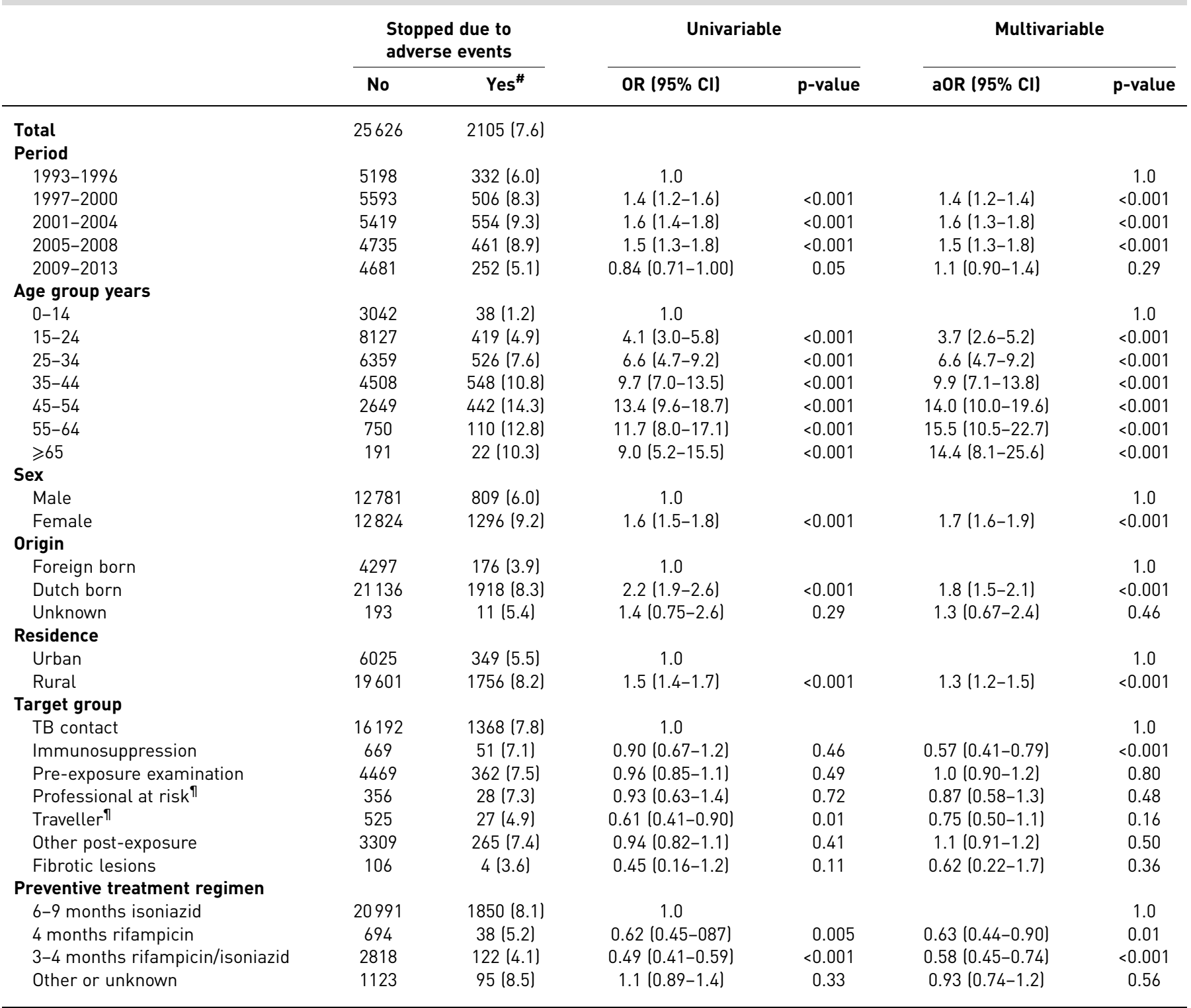

Data are presented as $\mathrm{n}$ or $\mathrm{n}(\%)$, unless otherwise stated. OR: odds ratio; aOR: adjusted OR; TB: tuberculosis. ${ }^{\#}$ : percentage stopped of row total given in parentheses; ${ }^{\text {१! }}$ post-exposure.

investigation. Target groups and demographic characteristics of cases changed over time, with more cases with immunosuppressive disorders, older cases and foreign-born cases notified in the last decade. Preventive treatment initiation was highest in groups more likely to progress to active TB: immunosuppressed persons, children and TB contacts. Children and immunosuppressed persons were also more likely to complete preventive treatment, as were persons treated with the rifampicin or rifampicin/isoniazid regimens. Those treated with these regimens were also less likely to stop preventive treatment due to adverse events.

The policy changes over time influenced annual rates of preventive treatment initiation and completion in various ways. Expansion of LTBI screening to older age and foreign-born groups reduced preventive treatment acceptance and preventive treatment completion, but the overall rates were likely mitigated by the introduction of immunosuppressed persons as a target group that showed relatively high rates of preventive treatment acceptance and completion.

The short-course treatment regimens of 3-4 months of rifampicin/isoniazid and 4 months of rifampicin were rapidly adopted since introduction in the Netherlands in 2009. The relatively high treatment completion rates are comparable to those reported among close contacts [8]. A recent systematic review of 
randomised clinical trials showed similar effectiveness and significantly less adverse events of rifampicin monotherapy and of the rifapentine/isoniazid regimen compared with isoniazid monotherapy [9]. In this study, the occurrence of adverse events for 3-4 months of rifampicin/isoniazid was not significantly different from those treated with isoniazid monotherapy, but the evidence used in this review was rated as very weak. In our observational study more persons receiving 6-9 months of isoniazid discontinued treatment because of adverse events. We conclude that in the Dutch setting, until the 12-dose rifapentine/ isoniazid regimen becomes available, the other rifamycin-based regimens have better completion rates and are thus to be preferred over the regimens with isoniazid alone.

Our study has a number of limitations. Registration is not mandatory, and in the course of the 21 years covered eligible LTBI cases for reporting have been both under-and overreported for the reasons explained above. Trends in preventive treatment initiation are therefore difficult to interpret. However, since 2005 the data have been used increasingly for management information purposes as well as surveillance, which may have improved accuracy, comparability and completeness. Therefore, we believe the treatment initiation rate over the period 2009-2013 is a reasonable reflection of the actual situation in the public health sector. Cases diagnosed and managed in the clinical sector, such as candidates for anti-TNF- $\alpha$ treatment, are increasingly reported since MPHSs have intensified collaboration with clinicians and provide treatment support to LTBI patients as well as TB patients identified in the clinical sector.

Another limitation when describing trends in preventive treatment initiation is the heterogeneity of the study population caused by policy changes over the years. For instance, until 2005, BCG-vaccinated persons and persons from high endemic areas or born in the Netherlands before 1945 (those with a high likelihood of remote infection) were not targeted for LTBI screening, not even in the context of contact investigation. Following the introduction of IGRA and the European consensus statement for contact investigation in 2010 [10], close contacts of infectious TB patients were included as a target group for LTBI testing irrespective of age, BCG vaccination status or country of origin. Some MPHSs were quick to adopt the new guidelines and others took longer. Such differences are difficult to avoid, because public health in the Netherlands is the responsibility of the local authority. Evidence-based and best-practice guidelines have been developed by the Committee for Practical TB Control Netherlands (CPT) on a consensus basis for more than 60 years, but the CPT has no mandate to demand full implementation.

The Netherlands is one of the few European countries monitoring and evaluating programmatic LTBI management [11]. A particular strength of the Dutch LTBI register is that it is integrated with the web-based TB surveillance system, which has a long-standing reputation for completeness and reliability [12]. However, to enable the evaluation of programmatic LTBI management, the present system needs adjustments so as to properly distinguish clinical target groups, reasons for not initiating preventive treatment, and the occurrence and nature of adverse events. Furthermore, the system does not record chemoprophylaxis for vulnerable populations (child TB contacts and immunocompromised TB contacts). This is an important intervention to prevent (serious) TB in these groups, which requires monitoring and evaluation as well. A revision of the CRF encompassing the variables listed above is planned for 2016.

The estimated risk reduction of preventive treatment for active TB among the population identified with LTBI was between $40 \%$ and $60 \%$. However, a high rate of acceptance and coverage of LTBI screening in the eligible target groups is also required for optimal impact of programmatic LTBI management. Information about the denominator of the target groups for LTBI and the number of persons per target group screened for LTBI have to be retrieved from other, generally not readily available sources. For TB contacts in the Netherlands, the information has been available through the TB register since 2006. For each notified TB patient it records whether a contact investigation was performed, how many contacts were eligible for screening, how many were screened and how many were identified with TB or LTBI [13]. In the period $2006-2010,87 \%$ of contacts eligible for screening were screened for TB, $73 \%$ were screened for LTBI and of those, $7 \%$ tested positive for LTBI [14].

\section{Conclusion}

The WHO End TB strategy calls upon low TB burden countries to move towards TB elimination by targeting high-risk TB groups for LTBI screening and preventive treatment [4]. The example of the Netherlands shows that given the right infrastructure and organisation of services, such groups can be successfully reached and served. Committed to the WHO End TB objective of $50 \%$ reduction of TB incidence by 2035, the Netherlands is ready to face the next challenge and explore the feasibility of targeting new migrants from high TB endemic countries for LTBI screening and preventive treatment in the coming years. Our study shows that when a high coverage and treatment acceptance can be achieved, a reduction of incident $\mathrm{TB}$ cases of $40-60 \%$ among new migrants is feasible. A flexible and sound TB surveillance system incorporating LTBI management is key to providing the basic indicators required for evaluation. 


\section{References}

1 Uplekar M, Weil D, Lonnroth K, et al. WHO’s new End TB Strategy. Lancet 2015; 385: 1799-1801.

2 Lonnroth K, Migliori GB, Abubakar I, et al. Towards tuberculosis elimination: an action framework for low-incidence countries. Eur Respir J 2015; 45: 928-952.

3 Diel R, Loddenkemper R, Zellweger JP, et al. Old ideas to innovate tuberculosis control: preventive treatment to achieve elimination. Eur Respir J 2013; 42: 785-801.

4 Getahun H, Matteelli A, Abubakar I, et al. Management of latent Mycobacterium tuberculosis infection: WHO guidelines for low tuberculosis burden countries. Eur Respir J 2015; 46: 1563-1576.

5 Erkens C, Slump E, Kamphorst M, et al. Coverage and yield of entry and follow-up screening for tuberculosis among new immigrants. Eur Respir J 2008; 32: 153-161.

6 Erkens CG, Dinmohamed AG, Kamphorst M, et al. Added value of interferon-gamma release assays in screening for tuberculous infection in the Netherlands. Int J Tuberc Lung Dis 2014; 18: 413-420.

7 World Health Organization. Guidelines on the Management of Latent Tuberculosis Infection. Geneva, World Health Organization, 2015.

8 Fiske CT, Yan F, Hirsch-MovermanY, et al. Risk factors for treatment default in close contacts with latent tuberculous infection. Int J Tuberc Lung Disy 2014; 18: 421-427.

9 Stagg HR, Zenner D, Harris RJ, et al. Treatment of latent tuberculosis infection: a network meta-analysis. Ann Intern Med 2014; 161: 419-428.

10 Erkens CG, Kamphorst M, Abubakar I, et al. Tuberculosis contact investigation in low prevalence countries: a European consensus. Eur Respir J 2010; 36: 925-949.

11 D'Ambrosio L, Dara M, Tadolini M, et al. Tuberculosis elimination: theory and practice in Europe. Eur Respir J 2014; 43: 1410-1420.

12 van Hest NA, Smit F, Baars HW, et al. Completeness of notification of tuberculosis in The Netherlands: how reliable is record-linkage and capture-recapture analysis? Epidemiol Infect 2007; 135: 1021-1029.

13 Mulder C, van Deutekom H, Huisman EM, et al. Coverage and yield of tuberculosis contact investigations in the Netherlands. Int J Tuberc Lung Dis 2011; 15: 1630-1637.

14 Rest JV, Erkens C, Vries GD. Evaluatie Bron- en Contactonderzoek bij Tuberculosepatiënten in Nederland, 2006-2010. [Evaluation of Source and Contact Investigation around Tuberculosis Patients in the Netherlands, 2006-2010.] The Hague, KNCV Tuberculosis Foundation, 2014. 